\title{
Subcutaneous adipose tissue plays a beneficial effect on subclinical atherosclerosis in young survivors of acute lymphocytic leukemia
}

This article was published in the following Dove Press journal:

Vascular Health and Risk Management

18 August 2015

Number of times this article has been viewed

\author{
Adriana Aparecida \\ Siviero-Miachon ${ }^{1,2}$ \\ Angela Maria \\ Spinola-Castro ${ }^{1,2}$ \\ Maria Lucia de \\ Martino Lee ${ }^{2}$ \\ Carlos Manoel \\ de Castro Monteiro 3 \\ Antonio Carlos de \\ Camargo Carvalho 4 \\ Antonio Ramos Calixto 5 \\ Bruno Geloneze ${ }^{5}$ \\ Gil Guerra-Junior ${ }^{6}$ \\ 'Division of Pediatric Endocrinology, \\ Department of Pediatrics, Federal \\ University of Sao Paulo (UNIFESP/ \\ EPM), ${ }^{2}$ Pediatric Oncology Institute - \\ IOP/GRAACC, Federal University of \\ Sao Paulo (UNIFESP/EPM), ${ }^{3}$ Private \\ Office, Castro Monteiro, Sao Paulo, \\ ${ }^{4}$ Division of Cardiology, Federal \\ University of Sao Paulo (UNIFESP/ \\ EPM), ${ }^{5}$ Laboratory of Investigation on \\ Metabolism and Diabetes (LIMED), \\ Faculty of Medical Sciences, State \\ University of Campinas (UNICAMP), \\ ${ }^{6}$ Division of Pediatric Endocrinology, \\ Department of Pediatrics, Faculty of \\ Medical Sciences, State University of \\ Campinas (UNICAMP), Brazil
}

Correspondence: Angela Maria Spinola-Castro

307 Doutor Diogo de Faria Street, 04037-000 Sao Paulo, Brazil

Tel +55 II 55799409

Fax +55 II 55799409

Email aspinola.dped@epm.br
Purpose: The aim of this study was to evaluate the relationship between body composition, metabolic profile, adipokines, and carotid intima-media thickness (cIMT) in young survivors of childhood acute lymphocytic leukemia (ALL).

Patients and methods: This cross-sectional study compared 55 ALL survivors, of chronological age between 15 years and 24 years, assigned into two groups according to the exposure to cranial radiation therapy (CRT; 25 irradiated and 30 nonirradiated) with 24 leukemia-free controls, and assessed body fat mass (dual-energy X-ray absorptiometry), computed tomography scan-derived abdominal adipose tissue, lipid profile, blood pressure (BP), adipokines, and cIMT by a multiple regression analysis.

Results: Treatment with CRT had an effect on all of the variables derived from the computed tomography scan: visceral adipose tissue (VAT) and subcutaneous adipose tissue (SAT) $(P<0.050)$. In a multiple linear regression model, cIMT positively correlated with exposure to CRT $(P=0.029)$, diastolic BP $(P=0.016)$, and leptin-to-adiponectin ratio $(P=0.048)$, while negatively related to SAT $(P=0.007)$.

Conclusion: In young survivors of childhood ALL, CRT modified the distribution of fat and played a critical role in determining cIMT. Leptin-to-adiponectin ratio, a biomarker of abdominal obesity and metabolic syndrome, and diastolic BP also influenced cIMT, a marker of subclinical atherosclerosis. Nonetheless, adiposity-associated vascular disease might be attenuated by SAT. Changes in body fat must be evaluated in this group of patients in the early course of survivorship in order to avoid premature cardiovascular disease associated with atherosclerosis. Yet, further research as regards the possible protective effect of SAT on vascular disease is warranted.

Keywords: precursor cell lymphoblastic leukemia-lymphoma/radiotherapy, abdominal fat, metabolic syndrome $\mathrm{X}$, adipokines, endothelium, atherosclerosis

\section{Introduction}

Acute lymphocytic leukemia (ALL) is the most prevalent type of cancer in childhood. ${ }^{1}$ As an increasing number of cancer survivors reaches adulthood, there may be consequences of the treatment, especially adiposity and components of the metabolic syndrome (MS), which predispose to cardiovascular disease (CVD) and enhance mortality. ${ }^{2-10}$ According to the recommendations of the American Association of Cardiology in collaboration with the Council for Cardiovascular Disease in the Young, ${ }^{3}$ based on the pediatric conditions and their risk of CVD, patients treated for childhood cancer should be classified as tier III, which means the presence of clinical criteria for MS and increased epidemiological risk of premature CVD. ${ }^{2-10}$ 
The pathophysiology of atherosclerosis involves many factors, such as endothelial dysfunction and chronic inflammation of the arteries. ${ }^{11,12}$ Insulin resistance (IR), hyperinsulinemia, and increased fatty free acids are considered the initial mechanisms of endothelial dysfunction, which progress to atherosclerosis, ${ }^{12,13}$ being worsened by other aspects, such as dyslipidemia, smoking, diabetes mellitus, and hypertension. ${ }^{11,12}$ Subclinical atherosclerosis may be detected by the increment of carotid intima-media thickness (cIMT), a simple and noninvasive method, which encompasses an intermediate marker of CVD. ${ }^{14-18}$

Adipokines, comprising adiponectin, leptin, resistin, cytokines, and monocyte chemoattractant protein-1 (MCP1), are also associated with endothelial dysfunction and atherogenesis. Adiponectin, as well as leptin-to-adiponectin ratio, is related to cIMT, and is considered a reliable indicator for atherosclerosis. ${ }^{19,20}$ In addition, the upregulation of endothelial cell MCP-1 production by low-density lipoprotein (LDL) oxidation is considered a major initiating event in atherogenesis. ${ }^{21}$

In cancer survivors, the risk factors for vascular structural and functional modifications include those related to the patient, disease, or treatment, especially chemotherapy and cranial radiation therapy (CRT) and/or cervical radiation therapy, but also hormonal deficiencies and the association with the components of the MS..$^{4,14-18}$

Nonetheless, there are few studies as regards the relationship between ALL, the exposure to CRT, the components of the MS, and the endothelium structure, ${ }^{2,8}$ and there is no study evaluating the adipokines in this context. Thus, the aim of this study was to evaluate the effect of body composition, metabolic profile, and adipokines on cIMT in young survivors of childhood ALL.

\section{Materials and methods}

\section{Study population}

This was a comparative cross-sectional study of a randomly selected sampling of ALL survivors of both sexes from the Pediatric Oncology Institute (Federal University of Sao Paulo, Brazil), admitted from May 1991 to June 2003, and a leukemia-free control group. The study was approved by the Ethics Research Committee of Federal University of Sao Paulo (Number 1197/07). To participate in this study, patients or parents, when appropriate, signed an informed consent form.

ALL subjects completed the Brazilian Cooperative Group for Treatment of Childhood Acute Lymphocytic
Leukemia (GBTLI) international protocol. Details comprising drugs and CRT can be read elsewhere..$^{22,23}$ CRT was applied prophylactically to those at high risk of relapse, defined according to the recommendations of the GBTLI, and/or therapeutically to those with central nervous system involvement, defined as blast cells greater than 5\% in cerebrospinal fluid..$^{22,23}$

The inclusion criteria comprised the following: chronological age between 15 years and 24 years; the complete clinical remission of ALL (complete absence of the disease in bone marrow and blood); no ALL therapy for at least 2 years; complete pubertal development (menarche in girls and Tanner stage IV or above in boys); ${ }^{24,25}$ growth of less than $1 \mathrm{~cm} /$ year; bone age with full epiphyseal plate fusion; normal renal, thyroid, gonadal, and adrenal profiles (spontaneous or under hormonal replacement therapy); and insulin-like growth factor-1 (IGF-1) within the normal range. The following were excluded from the study: patients who used anorexigens, insulin-sensitivity medications, or other drugs that interfere with adiposity (eg, metformin, sibutramine, and fluoxetine); those who had experienced exogenous growth hormone $(\mathrm{GH})$ administration within 2 years prior to study enrollment or a bone marrow transplantation; and those who were pregnant, were postpartum, or had Down's syndrome.

Survivors were stratified into two groups according to the exposure to CRT (irradiated and nonirradiated). Characteristics of host/disease and therapy were assessed from clinical examinations and/or medical records, encompassing sex, age at ALL diagnosis, age at assessment, age at the time of receiving and dose of CRT (if employed), time post-therapy, medications, or eventful past medical history.

The control group was selected among peers and/or relatives of the ALL patients, and was composed of healthy, leukemia-free subjects of similar age and body mass index (BMI), who did not use any medication.

\section{Variables}

All the variables were determined for both ALL patients (irradiated or not) and the control group.

\section{Adiposity indexes}

Adiposity indexes comprised body composition and fat distribution variables. Body composition encompassed BMI and fat mass index (FMI). BMI was calculated as the weight in kilograms divided by height in meters squared, and converted to $Z$ scores based on the National Center 
for Health Statistics 2000 Centers for Disease Control and Prevention growth charts. ${ }^{26}$ The FMI was calculated as the fat mass (in kilograms) divided by height in meters squared $\left(\mathrm{kg} / \mathrm{m}^{2}\right)$, body fat mass being assessed by dual-energy X-ray absorptiometry, Hologic Discovery 4500 (QDR-4500A; Hologic Inc., Bedford, MA, USA).

Fat distribution indexes comprised computed tomography (CT) scan-derived abdominal adipose tissue, encompassing visceral adipose tissue (VAT) and subcutaneous adipose tissue (SAT), both measured according to the method described elsewhere. ${ }^{6,9}$

\section{Blood pressure, metabolic profile, and growth factor} Systolic blood pressure (BP) and diastolic BP (in $\mathrm{mmHg}$ ) were both measured with the patient in the sitting position after 5 minutes of rest in a tranquil environment, by auscultation, at an average of three outlets in the right arm $\left(\right.$ Tycos ${ }^{\circledR}$ Welch Allyn, NY, USA).

Metabolic profile included LDL cholesterol (LDL-C), high-density lipoprotein cholesterol (HDL-C), and triglycerides (enzymatic colorimetric method, in mmol/L). Glucose values (method: automated) and insulin (method: ACTIVE $^{\circledR}$ Insulin ELISA DSL-10-1600; Diagnostics Systems Laboratories, Inc., Webster, TX, USA) were used to calculate the homeostasis model assessment - insulin resistance (HOMA1-IR). ${ }^{27}$

IGF-1 (in nmol/L) was analyzed by a sandwich assay, one-step chemiluminescence using coated magnetic microparticles (LIAISON ${ }^{\circledR}$ IGF-1 OS-313, 231; Gruppe Biomedica, Inc., Vienna, Austria), whose intra-assay coefficient of variability $(\mathrm{CV})$ and inter-assay $\mathrm{CV}$ were, respectively, $3.7 \%$ and $4.6 \%$, with minimum detection limit of $3.0 \mathrm{ng} / \mathrm{mL}$ and $100 \%$ of specificity.

\section{Adipokines}

1. Leptin-to-adiponectin ratio $(\mathrm{ng} / \mu \mathrm{g})$ : leptin (in $\mathrm{ng} / \mathrm{mL}$ ) being analyzed by "Dual Range" enzyme-linked immunosorbent assay (ELISA) (Linco Research, Inc., St Charles, MO, USA; catalog number EZHL-80SK) with intraassay $\mathrm{CV}$ of $3.6 \%$, inter-assay $\mathrm{CV}$ of $4.0 \%$, minimum detection limit of $0.5 \mathrm{ng} / \mathrm{mL}$, and specificity of $100 \%$, and adiponectin (in $\mu \mathrm{g} / \mathrm{mL}$ ) being evaluated by ELISA (R\&D Systems, Inc., Minneapolis, MN, USA; catalog number DY1065) with intra-assay CV of $6.2 \%$, inter-assay CV of $7.2 \%$, minimum detection limit of $0.1 \mathrm{ng} / \mathrm{mL}$, and specificity of $100 \%$.

2. MCP-1 (in pg/mL): ELISA (R\&D Systems, Inc.; cata$\log$ number DY279) with intra-assay CV below 10\%, inter-assay $\mathrm{CV}$ below 15\%, minimum detection limit of $10 \mathrm{pg} / \mathrm{mL}$, and specificity of $100 \%$.

\section{Carotid intima-media thickness}

cIMT was measured in the supine position, $1.0 \mathrm{~cm}$ below the carotid bulb of right and left common carotid arteries $\left(\right.$ Sonos 5500, Hewlett Packard ${ }^{\circledR}$ ), coupled to a high-resolution linear transducer with frequency up to $11 \mathrm{MHz}$, and image acquisition was performed in the longitudinal plane in twodimensional mode, with simultaneous cardiac monitoring, by one of two experienced observers, whose intraobserver variation was $0.05 \pm 0.02 \mathrm{~mm}$ and interobserver variation was $0.07 \pm 0.04 \mathrm{~mm}$. The measurement was performed in the anterior or posterior wall of the artery, in the distance between two lines represented by the echogenic interfaces lumen-intima and media-adventitia of carotid wall. For each side (right and left), the result was calculated by the average of the measurements of three consecutive cardiac cycles, taking into consideration the $\mathrm{R}$ wave peak. ${ }^{28}$ Subsequently, the mean measurement of both sides was used for further analyses: (right cIMT + left cIMT)/2.

\section{Statistical analysis}

Statistical analysis was initially made in a descriptive way through some summary measures such as mean, median, minimum and maximum values, standard deviation (SD), and absolute and relative frequencies (percentage). The inferential analyses were the following:

- As regards exposure to CRT/group (nonirradiated ALL patients vs irradiated ALL patients vs control subjects): Kruskal-Wallis test, with Bonferroni-Dunn post hoc test for pairwise comparisons if statistically significant, in the comparison of age at assessment (years), BMI ( $Z$ score), VAT $\left(\mathrm{cm}^{3}\right)$, SAT $\left(\mathrm{cm}^{3}\right)$, HOMA1-IR, triglycerides (mmol/L), systolic and diastolic BP (mmHg), IGF-1 $(\mathrm{nmol} / \mathrm{L})$, leptin-to-adiponectin ratio $(\mathrm{ng} / \mu \mathrm{g}), \mathrm{MCP}-1$ ( $\mathrm{pg} / \mathrm{mL})$, and cIMT (mm); one-way analysis of variance, with Duncan post hoc test for pairwise comparisons if statistically significant, in the comparison of FMI $\left(\mathrm{kg} / \mathrm{m}^{2}\right)$, LDL-C (mmol/L), and HDL-C (mmol/L); and Student's $t$-test for independent samples in the comparison of time post-therapy (years) between ALL subjects.

- As regards sex of ALL patients: Mann-Whitney test in the comparison of age at assessment (years), BMI ( $Z$ score), FMI $\left(\mathrm{kg} / \mathrm{m}^{2}\right)$, VAT $\left(\mathrm{cm}^{3}\right)$, SAT $\left(\mathrm{cm}^{3}\right)$, HOMA1-IR, triglycerides $(\mathrm{mmol} / \mathrm{L})$, diastolic BP $(\mathrm{mmHg}), \mathrm{IGF}-1$ $(\mathrm{nmol} / \mathrm{L})$, leptin-to-adiponectin ratio $(\mathrm{ng} / \mu \mathrm{g}), \mathrm{MCP}-1$ $(\mathrm{pg} / \mathrm{mL})$, and cIMT $(\mathrm{mm})$; and Student's $t$-test for 
independent samples in the comparison of time posttherapy (years), LDL-C (mmol/L), HDL-C (mmol/L), and systolic BP (mmHg).

- As regards sex of the control group: Mann-Whitney test in the comparison of FMI $\left(\mathrm{kg} / \mathrm{m}^{2}\right)$, VAT $\left(\mathrm{cm}^{3}\right)$, SAT $\left(\mathrm{cm}^{3}\right)$, HOMA1-IR, triglycerides $(\mathrm{mmol} / \mathrm{L})$, systolic BP (mmHg), and cIMT (mm); and Student's $t$-test for independent samples in the comparison of age at assessment (years), BMI (Z score), LDL-C (mmol/L), HDL-C $(\mathrm{mmol} / \mathrm{L})$, diastolic BP (mmHg), IGF-1 (nmol/L), leptinto-adiponectin ratio $(\mathrm{ng} / \mu \mathrm{g})$, and MCP-1 (pg/mL).

- Multiple linear regression analysis to study the relationship of cIMT (mm) according to the exposure to CRT, VAT $\left(\mathrm{cm}^{3}\right)$, SAT $\left(\mathrm{cm}^{3}\right)$, LDL-C $(\mathrm{mmol} / \mathrm{L})$, HDL-C $(\mathrm{mmol} / \mathrm{L})$, triglycerides $(\mathrm{mmol} / \mathrm{L})$, systolic and diastolic BP (mmHg), leptin-to-adiponectin ratio ( $\mathrm{ng} / \mu \mathrm{g})$, and MCP-1 (pg/mL), among ALL subjects.

- To further explore the relation between VAT and SAT among ALL patients, the VAT-to-SAT ratio was determined, and the relationship between this ratio and cIMT was evaluated by Pearson's correlation.

Statistical analyses were performed with the Statistical Package for the Social Sciences (SPSS) version 19.0 for Windows and $\mathrm{R}$ version 2.15.2. In all tests, $P<0.050$ was considered as statistically significant.

\section{Results}

\section{Subject's characteristics}

The selected sample in this study consisted of 79 subjects, 55 being (69.6\%) ALL patients, and 24 (30.4\%), controls.

The age at assessment of the ALL patients was (given as mean with SD in parentheses) 18.6 (2.5) years, ranging from 15.0 years to 22.9 years, age at ALL diagnosis was 7.4 (3.9) years, and time post-therapy was 8.5 (3.5) years. Controls were 19.8 (3.1) years at study assessment, ranging from 14.3 years to 24.9 years. While compared to nonirradiated ALL subjects, controls were older (post hoc test, $P=0.049$ ) (Table 1).

The ALL subjects were composed of 31 (56.4\%) women and $24(43.6 \%)$ men. The control group comprised 14 (58.3\%) women and $10(41.7 \%)$ men.

ALL subjects completed the GBTLI international protocol, and 25 (45.4\%) ALL survivors received CRT with a dose of either $18 \mathrm{~Gy}(76 \%)$ or $24 \mathrm{~Gy}(24 \%)$ at a mean age of 8.0 (4.0) years. Three subjects also received alternative protocols to the GBTLI, which were employed due to medullar or testicular relapses but without complementary CRT. Among the irradiated survivors, one subject also received spinal irradiation, and two patients received testicular radiation therapy (24 Gy).

\section{Body composition, fat distribution, and cIMT}

FMI was increased in female sex in both groups of patients, ALL subjects and controls $(P<0.050)$, and showed a trend toward an increase in ALL patients subjected to CRT $(P=0.078)$. (Table 1 presents the comparison with regard to CRT exposure/group, and Table 2 presents further data as regards sex between ALL subjects.) Data regarding sex among controls are not shown.

VAT (post hoc test, $P=0.018$ ) and SAT (post hoc test, $P=0.050$ ) were increased among irradiated compared to nonirradiated ALL subjects, with no difference while compared to the controls. Concerning sex, no differences in VAT or SAT were detected in either ALL subjects or controls (Tables 1 and 2).

cIMT showed no difference as regards CRT exposure/ group or sex, in both ALL subjects and controls (Tables 1 and 2).

\section{Metabolic profile, adipokines, and BP}

While analyzing data regarding the three groups (Table 1), LDL-C was increased in both irradiated ALL patients and controls, when compared to nonirradiated ALL patients (post hoc test, $P<0.050$ ), and MCP-1 was increased in controls $(P=0.046)$, but no significant difference was determined in a post hoc test analysis.

When comparing sexes among ALL patients (Table 2), HDL-C was decreased $(P=0.006)$, while both systolic BP $(P=0.028)$ and leptin-to-adiponectin ratio $(P=0.011)$ were increased in ALL males.

There were no other statistically significant differences with respect to other characteristics measured and shown in Table 1 or 2, or regarding sex among the control group (data not shown).

\section{Multiple linear regressions}

This analysis was performed among ALL patients and showed that cIMT was positively related to the exposure to CRT $(P=0.029)$, diastolic BP $(P=0.016)$, and leptin-toadiponectin ratio $(P=0.048)$, while negatively related to SAT $(P=0.007)$.

In the presence of exposure to CRT, SAT, diastolic BP and leptin-to-adiponectin ratio, VAT $(P=0.437)$, LDL-C $(P=0.252)$, HDL-C $(P=0.411)$, triglycerides $(P=0.390)$, systolic BP $(P=0.977)$, and MCP-1 $(P=0.741)$ did not influence cIMT. Table 3 presents model adjustment. 


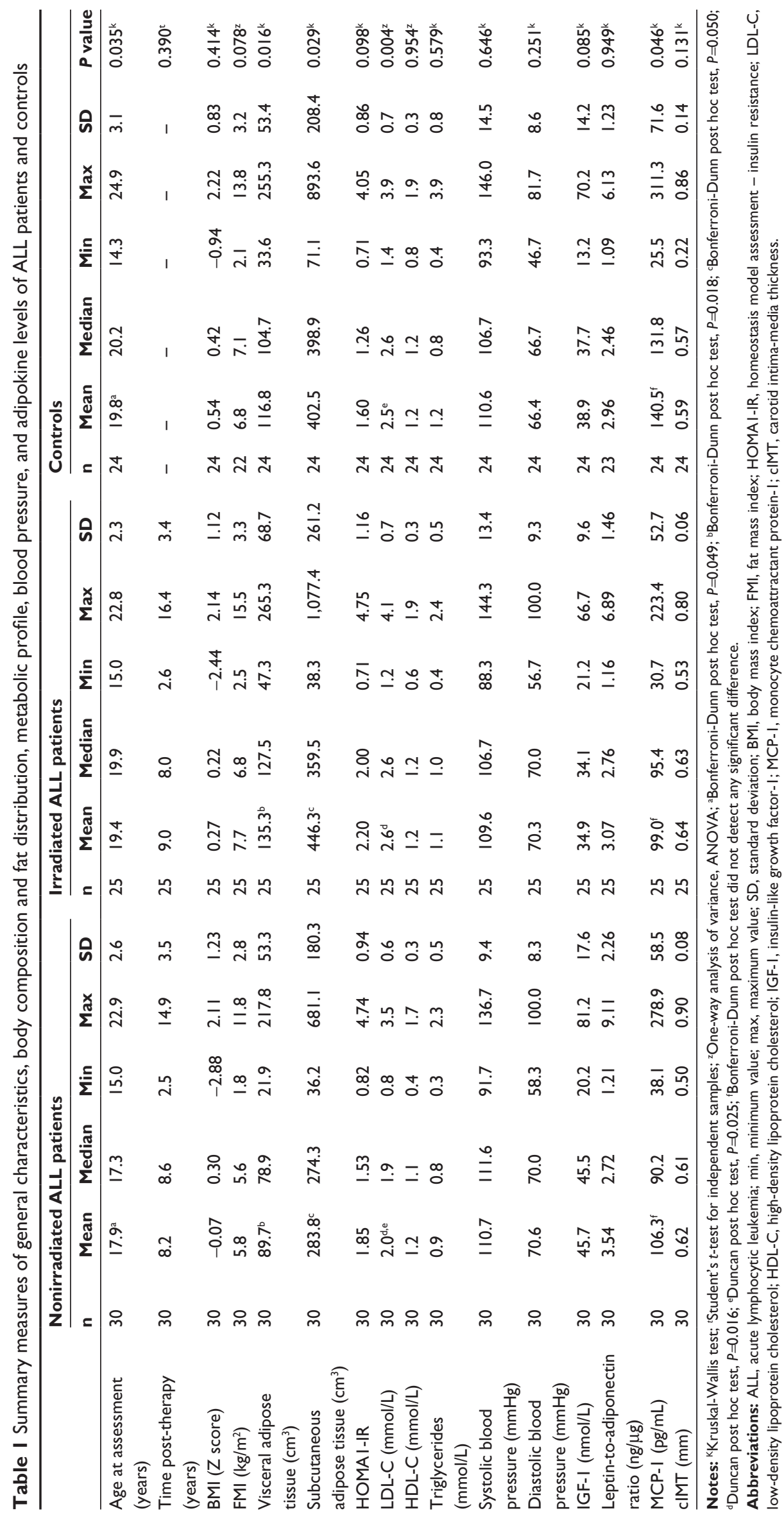


Table 2 Summary measures of general characteristics, body composition and fat distribution, metabolic profile, blood pressure, and adipokine levels of ALL patients, according to sex

\begin{tabular}{|c|c|c|c|c|c|c|c|c|c|c|c|c|c|}
\hline & \multicolumn{13}{|c|}{ Sex } \\
\hline & \multicolumn{6}{|c|}{ Female } & \multicolumn{7}{|c|}{ Male } \\
\hline & $\mathbf{n}$ & Mean & Median & Min & Max & SD & $\mathbf{n}$ & Mean & Median & Min & Max & SD & $P$ value \\
\hline $\begin{array}{l}\text { Age at assessment } \\
\text { (years) }\end{array}$ & 31 & 18.4 & 18.1 & 15.0 & 22.9 & 2.7 & 24 & 18.9 & 18.6 & 15.0 & 22.8 & 2.3 & $0.40 \mathrm{I}^{\mathrm{m}}$ \\
\hline $\begin{array}{l}\text { Time post-therapy } \\
\text { (years) }\end{array}$ & 31 & 8.5 & 8.0 & 4.0 & 16.4 & 3.5 & 24 & 8.5 & 8.4 & 2.5 & 14.3 & 3.5 & $0.968^{\mathrm{t}}$ \\
\hline BMI (Z score) & 31 & 0.15 & 0.26 & -2.55 & 1.96 & 1.04 & 24 & 0.00 & 0.30 & -2.88 & 2.14 & 1.36 & $0.665^{\mathrm{m}}$ \\
\hline $\mathrm{FMl}\left(\mathrm{kg} / \mathrm{m}^{2}\right)$ & 31 & 7.8 & 7.3 & 3.2 & 15.5 & 2.8 & 24 & 5.2 & 4.3 & 1.8 & 11.8 & 3.1 & $0.00 \mathrm{I}^{\mathrm{m}}$ \\
\hline $\begin{array}{l}\text { Visceral adipose } \\
\text { tissue }\left(\mathrm{cm}^{3}\right)\end{array}$ & 31 & 99.3 & 81.3 & 24.0 & 250.9 & 59.1 & 24 & 124.7 & 100.6 & 21.9 & 265.3 & 69.2 & $0.149^{m}$ \\
\hline $\begin{array}{l}\text { Subcutaneous } \\
\text { adipose tissue }\left(\mathrm{cm}^{3}\right)\end{array}$ & 31 & 386.1 & 359.5 & 96.9 & $\mathrm{I}, 077.4$ & 205.2 & 24 & 320.9 & 270.1 & 36.2 & 934.2 & 265.3 & $0.096^{m}$ \\
\hline HOMAI-IR & 31 & 2.00 & 1.63 & 0.80 & 4.75 & 1.00 & 24 & 2.03 & 1.62 & 0.71 & 4.49 & 1.13 & $0.859^{m}$ \\
\hline LDL-C (mmol/L) & 31 & 2.3 & 2.3 & I.I & 3.5 & 0.7 & 24 & 2.2 & 2.0 & 0.8 & 4.1 & 0.8 & $0.545^{\mathrm{t}}$ \\
\hline HDL-C (mmol/L) & 31 & 1.3 & 1.3 & 0.4 & 1.9 & 0.3 & 24 & I.I & I.I & 0.6 & 1.4 & 0.2 & $0.006^{t}$ \\
\hline $\begin{array}{l}\text { Triglycerides } \\
(\mathrm{mmol} / \mathrm{L})\end{array}$ & 31 & 1.0 & 0.9 & 0.3 & 2.4 & 0.6 & 24 & 1.0 & 0.8 & 0.5 & 1.9 & 0.5 & $0.939^{m}$ \\
\hline $\begin{array}{l}\text { Systolic blood } \\
\text { pressure }(\mathrm{mmHg})\end{array}$ & 31 & 107.3 & 106.7 & 90.0 & 122.0 & 8.9 & 24 & 114.0 & 113.3 & 88.3 & 144.3 & 13.0 & $0.028^{t}$ \\
\hline $\begin{array}{l}\text { Diastolic blood } \\
\text { pressure }(\mathrm{mmHg})\end{array}$ & 31 & 68.4 & 66.7 & 56.7 & 80.0 & 5.8 & 24 & 73.1 & 70.0 & 56.7 & 100.0 & 10.9 & $0.15 \mathrm{I}^{\mathrm{m}}$ \\
\hline IGF-I (nmol/L) & 31 & 39.9 & 34.7 & 20.6 & 70.5 & 15.4 & 24 & 41.8 & 36.2 & 20.2 & 81.2 & 15.7 & $0.530^{\mathrm{m}}$ \\
\hline $\begin{array}{l}\text { Leptin-to-adiponectin } \\
\text { ratio }(\mathrm{ng} / \mu \mathrm{g})\end{array}$ & 31 & 2.92 & 2.35 & 1.16 & 9.11 & 1.90 & 24 & 3.85 & 3.27 & 1.58 & 8.02 & 1.88 & $0.01 \mathrm{I}^{\mathrm{m}}$ \\
\hline MCP-I (pg/mL) & 31 & 101.5 & 92.1 & 45.8 & 198.3 & 44.9 & 24 & 104.9 & 88.5 & 30.7 & 278.9 & 67.8 & $0.64 \mathrm{I}^{\mathrm{m}}$ \\
\hline $\mathrm{clMT}(\mathrm{mm})$ & 31 & 0.61 & 0.63 & 0.50 & 0.78 & 0.06 & 24 & 0.64 & 0.62 & 0.53 & 0.90 & 0.09 & $0.463^{\mathrm{m}}$ \\
\hline
\end{tabular}

Notes: mMann-Whitney test; 'Student's t-test for independent samples.

Abbreviations: ALL, acute lymphocytic leukemia; min, minimum value; max, maximum value; SD, standard deviation; BMI, body mass index; FMI, fat mass index; HOMAIIR, homeostasis model assessment - insulin resistance; LDL-C, low-density lipoprotein cholesterol; HDL-C, high-density lipoprotein cholesterol; IGF-I, insulin-like growth factor-I; MCP-I, monocyte chemoattractant protein-I; cIMT, carotid intima-media thickness.

\section{VAT-to-SAT ratio}

cIMT and VAT-to-SAT ratio were positively correlated among ALL patients, as shown in Figure $1(r=0.355$, $P=0.008$ ).

\section{Discussion}

This study investigated the effect of fat distribution, lipid profile, BP, and adipokines on cIMT in young survivors of childhood ALL, and showed that even though CRT led to abdominal accumulation of fat and positively determined cIMT, a noninvasive assessment of subclinical atherosclerosis,
SAT might represent a protective factor against adiposityassociated vascular disease.

The increase in cIMT in some groups of cancer survivors, particularly patients with brain tumors and Hodgkin's lymphoma, has been explained by a local effect of CRT and/ or neck irradiation, which is dose-dependent, and influenced by other factors, such as chronological age, time since CRT, and association with components of the MS and GH insufficiency. ${ }^{410}$ An increase in cIMT has been observed in the group of patients who were treated with high doses of CRT (54 Gy) and/or cervical irradiation., ${ }^{4,14,16-18,29}$

Table 3 Estimated parameters from multiple linear regression model, with the dependent variable being carotid intima-media thickness, among ALL patients

\begin{tabular}{|c|c|c|c|c|}
\hline & Coefficient & Standard error (coefficient) & $t$ & $P$ value \\
\hline (Constant) & 0.420640 & $0.077 \mid 23$ & 5.454 & $<0.001$ \\
\hline Exposure to CRT & 0.044374 & 0.019753 & 2.246 & 0.029 \\
\hline $\operatorname{SAT}\left(\mathrm{cm}^{3}\right)$ & -0.000128 & 0.000045 & -2.830 & 0.007 \\
\hline Diastolic blood pressure $(\mathrm{mmHg})$ & 0.0028815 & 0.001131 & 2.488 & 0.016 \\
\hline Leptin-to-adiponectin ratio (ng/mcg) & 0.009745 & 0.004807 & 2.027 & 0.048 \\
\hline
\end{tabular}

Abbreviations: ALL, acute lymphocytic leukemia; CRT, cranial radiation therapy; SAT, subcutaneous adipose tissue. 


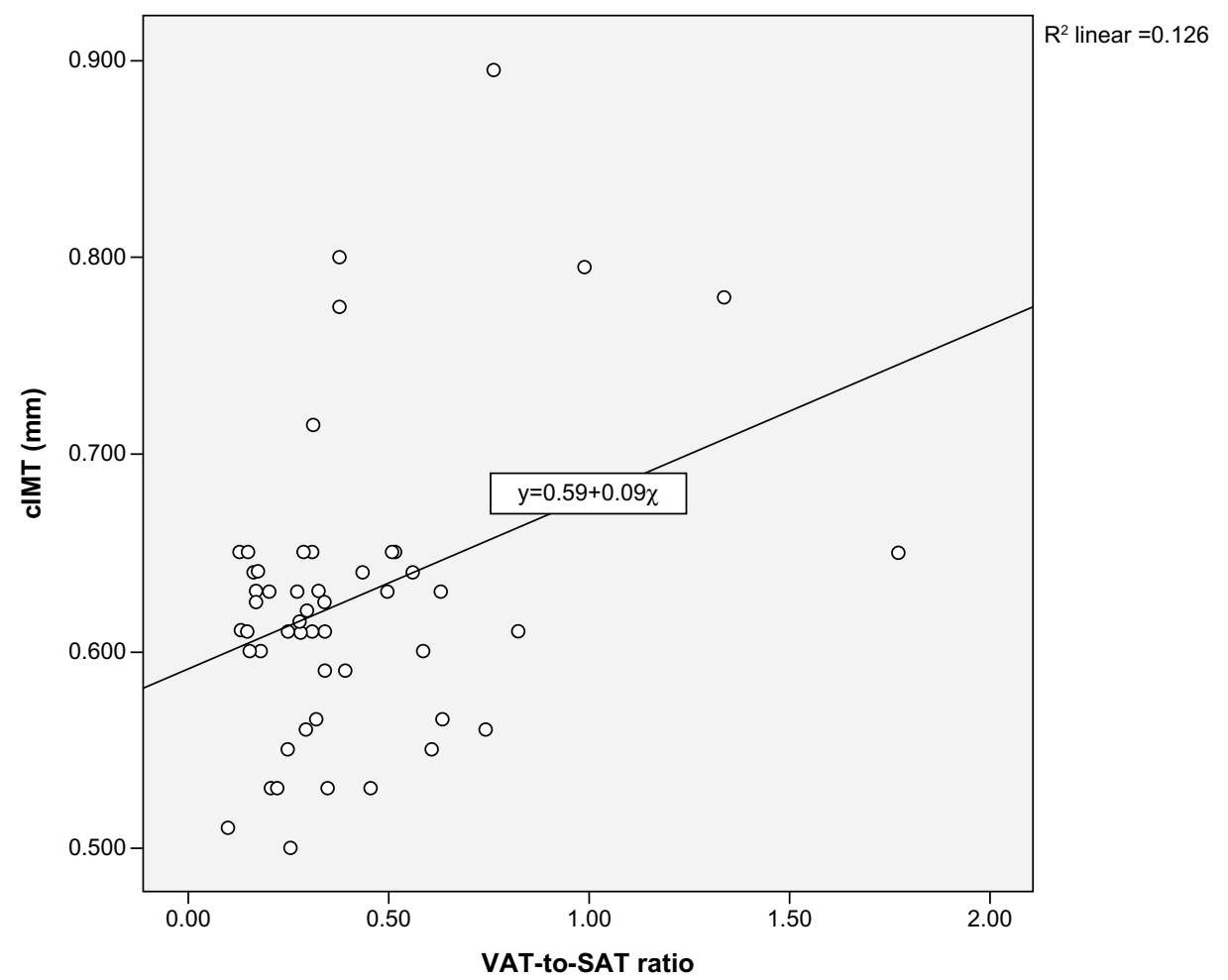

Figure I Scatterplot and trend line between carotid intima-media thickness and visceral-to-subcutaneous adipose tissue ratio, among ALL patients. Note: Pearson's correlation; $r=0.355, P=0.008$.

Abbreviations: ALL, acute lymphocytic leukemia; VAT, visceral adipose tissue; SAT, subcutaneous adipose tissue; cIMT, carotid intima-media thickness.

In the present study, there was no significant difference in cIMT, taking into account CRT treatment or sexes. This finding is in accordance with previous studies concerning ALL survivors who received either low doses of CRT or no radiation, either adult long-term survivors or those who had just received their treatment, survivors who did not show any increase in carotid vascular measures, while compared to normal controls. ${ }^{2,29}$ It should be noted, however, that contrary to brain tumor survivors, patients treated for ALL receive less radiation overall and not specifically to the neck region. One of these studies in ALL survivors demonstrated only vascular dysfunction (but not increase in cIMT), as a marker of subclinical atherosclerosis, which was not evaluated in the present sample of ALL survivors so far. ${ }^{29}$ Conversely, the present sample of ALL survivors showed an increase in cIMT, while compared to patients selected from the Brazilian Metabolic Syndrome Study, matched by sex, chronological age, and BMI ${ }^{30}$ Although age and sex are nonmodifiable elements that should be taken into account for vascular evaluation, ${ }^{31}$ to date, it is not possible to state that time off therapy, dose of CRT, sex, and chronological age have influenced the results in the present study, since patients were relatively young, evaluated with few time off therapy, and received low doses of CRT. ${ }^{4,14,16-18,29,31}$
CRT played a role in cIMT among this sample of young survivors of ALL, and might be considered a positive predictor of subclinical atherosclerosis, even though it is not possible to determine the critical dose of CRT associated with an increase in vascular structure so far. The exposure to CRT in ALL survivors caused a change in the distribution of fat, predominantly in the abdominal region, as previously documented in a study with this sample of ALL patients. ${ }^{6}$ Since the exact mechanism by which CRT modifies adipose tissue distribution is unknown, it is possible to declare that there was a relationship between the exposure to CRT and cIMT in this sample of ALL survivors. Excessive abdominal fat accumulation has been considered a frequent adverse effect in the period of the rebound of adiposity, and many years after therapy withdrawal in ALL survivors, particularly those exposed to CRT. CRT may affect energy regulatory pathways and the hypothalamic metabolic circuits resulting in body fat alterations. A hypothalamic insult, such as CRT or intrathecal methotrexate, may lead to modifications in satiety centers or increase parasympathetic tone. Moreover, GH deficiency, which might occur in ALL patients subjected to CRT, may have an impact on body composition and metabolic profile..$^{2-69,32}$

SAT was a negative predictor of cIMT in this sample of young survivors of ALL, which concurs with previous 
statements that have evaluated the relationship between VAT and SAT. ${ }^{33,34}$ Furthermore, the VAT-to-SAT ratio also played a positive role in cIMT among ALL subjects. It appears that there is an interaction of SAT by modifying adverse effects of VAT, or the adverse effects of VAT might be lessened with increasing amounts of SAT. Nonetheless, SAT seems to display less insulin- and adrenergic-related lipolytic activity, to release fewer inflammatory adipokines and to have greater adiponectin expression, compared to VAT. The mechanisms that may underlie the potential beneficial effects of SAT in the presence of high amounts of VAT are not clear so far..$^{33,34}$

Diastolic BP showed a positive effect on cIMT in this sample of patients treated for ALL, but there was no relationship between cIMT and HOMA1-IR or lipid profile, which concurs with previous studies, ${ }^{20}$ even though factors associated with MS, such as HDL-C, LDL-C, and IR, potentially aggravate endothelial dysfunction and atherogenesis. ${ }^{12,35,36} \mathrm{In}$ relation to hormonal deficiencies, especially GH deficiency, there were no statistical differences in IGF-1 levels while comparing groups, even though the group exposed to CRT showed lower levels, not clinically relevant and within the normal limits so far.

This study is the first to describe the relationship between cIMT, components of the MS, and adipokines in patients treated for ALL, either exposed or not to CRT. Leptin-toadiponectin ratio, which reflects abdominal fat accumulation and is a biomarker of the MS, was associated with cIMT in this sample of young survivors of ALL, which has been previously described. ${ }^{19,20}$ Even though oxidized LDL-C may increase adherence and penetration of macrophages in the endothelial cell, this is a local event, and might explain why MCP-1 levels were not related to cIMT in this study. ${ }^{21}$

This study included 55 survivors of ALL from a single institution, at a mean 8.5 years posttreatment, younger, at a shorter posttreatment interval compared to other studies, and none currently assessed within the period of the adiposity rebound, which features the 2 years after treatment withdrawal, in which there is an increment of the adiposity indexes..$^{2,5,8,9,32,37}$ The sample size was small, and this may be considered a limitation of the study. It is noteworthy, however, that even though some of the characteristics of the control group (age, FMI, lipid profile, and adipokines) resemble those of the irradiated ALL patients, VAT and SAT were probably increased by CRT exposure, considering that this sample of ALL patients received intense medical support, overprotection due to illness, and parental caregiving, therefore reinforcing the deleterious effect of CRT on abdominal accumulation of fat among ALL survivors. Patients not subjected to CRT were younger than leukemia-free controls; however, this difference regarding age was not clinically relevant. ALL patients subjected to CRT may have undergone preceding GBTLI protocols (GBTLI-85 and GBTLI-93), ${ }^{22,23}$ which indicated prophylactic CRT to all patients at risk of relapse. Concerning CRT and its detrimental effects, the latest GBTLI-99 protocol has reserved therapeutic CRT entirely to patients with central nervous system involvement so that patients treated for ALL are not exposed to prophylactic CRT so far. ${ }^{23}$ Another aspect to be emphasized is that this study did not evaluate the endothelium function but only structural alterations of carotid wall. Moreover, there are no data concerning cIMT and/or adipokines before or during ALL therapy.

\section{Conclusion}

In young survivors of childhood ALL, CRT modified the distribution of fat and was a positive predictor of cIMT. Leptinto-adiponectin ratio, a biomarker of abdominal obesity and the MS, and diastolic BP also determined cIMT, a marker of subclinical atherosclerosis. Nonetheless, adiposity-associated vascular disease might be attenuated by SAT. Changes in body fat must be evaluated in this group of patients in the early course of survivorship in order to avoid premature CVD associated with atherosclerosis. Yet, further research as regards the possible protective effect of SAT on vascular disease is warranted.

\section{Acknowledgments}

The authors greatly thank the patients and their families. The authors appreciate the support of Ricardo Silva Ribeiro for performing the CT scans and abdominal fat layer measurements, and the statistical revision of Gianni Santos and Mitti Koyama. This work was supported by grants from the Sao Paulo State Research Foundation (number 06/06162-9 to GG-J), and was presented, in part, at the 13th International Conference on Long-Term Complications of Treatment of Children and Adolescents for Cancer, which was held at Peabody Hotel in Memphis, TN, USA, in June 2013.

\section{Author contributions}

AAS-M participated in study design, data collection, and organization, as well as preparation of the manuscript. AMS-C also participated in study design and conception, preparation, and revision of the manuscript. MLML participated in data collection and organization, as well as in 
revision of the manuscript. BG, ARC, and GG-J oversaw the study, its design, and conception, and revised the manuscript. CMCM and ACCC performed all evaluations of cIMT and revised the manuscript.

\section{Disclosure}

The authors report no conflicts of interest in this work.

\section{References}

1. Redaelli A, Laskin BL, Stephens JM, Botteman MF, Pashos CL. A systematic literature review of the clinical and epidemiological burden of acute lymphoblastic leukaemia (ALL). Eur J Cancer Care (Engl). 2005;14:53-62.

2. Link K, Moëll C, Garwicz S, et al. Growth hormone deficiency predicts cardiovascular risk in young adults treated for acute lymphoblastic leukemia in childhood. J Clin Endocrinol Metab. 2004;89:5003-5012.

3. Kavey RE, Allada V, Daniels SR, et al; American Heart Association Expert Panel on Population and Prevention Science, American Heart Association Council on Cardiovascular Disease in the Young, American Heart Association Council on Epidemiology and Prevention, American Heart Association Council on Nutrition, Physical Activity and Metabolism, American Heart Association Council on High Blood Pressure Research, American Heart Association Council on Cardiovascular Nursing, American Heart Association Council on the Kidney in Heart Disease, Interdisciplinary Working Group on Quality of Care and Outcomes Research. Cardiovascular risk reduction in high-risk pediatric patients: a scientific statement from the American Heart Association Expert Panel on Population and Prevention Science; the Councils on Cardiovascular Disease in the Young, Epidemiology and Prevention, Nutrition, Physical Activity and Metabolism, High Blood Pressure Research, Cardiovascular Nursing, and the Kidney in Heart Disease; and the Interdisciplinary Working Group on Quality of Care and Outcomes Research: endorsed by the American Academy of Pediatrics. Circulation. 2006;114:2710-2738.

4. Siviero-Miachon AA, Spinola-Castro AM, Guerra-Junior G. Detection of metabolic syndrome features among childhood cancer survivors: a target to prevent disease. Vasc Health Risk Manag. 2008;4: 825-836.

5. Siviero-Miachon AA, Spinola-Castro AM, Guerra-Junior G. Adiposity in childhood cancer survivors: insights into obesity physiopathology. Arq Bras Endocrinol Metabol. 2009;53:190-200.

6. Siviero-Miachon AA, Spinola-Castro AM, Lee ML, et al. Cranial radiotherapy predisposes to abdominal adiposity in survivors of childhood acute lymphocytic leukemia. Radiat Oncol. 2013;8:39.

7. van Waas M, Neggers SJ, Pieters R, van den Heuvel-Eibrink MM. Components of the metabolic syndrome in 500 adult long-term survivors of childhood cancer. Ann Oncol. 2010;21:1121-1126.

8. Oeffinger KC, Buchanan GR, Eshelman DA, et al. Cardiovascular risk factors in young adult survivors of childhood acute lymphoblastic leukemia. J Pediatr Hematol Oncol. 2001;23:424-430.

9. Janiszewski PM, Oeffinger KC, Church TS, et al. Abdominal obesity, liver fat, and muscle composition in survivors of childhood acute lymphoblastic leukemia. J Clin Endocrinol Metab. 2007;92: 3816-3821.

10. Siviero-Miachon AA, Monteiro CM, Pires LV, et al. Early features of metabolic syndrome in pediatric post-cancer survivors: outcomes in adolescents and young adults treated for childhood medulloblastoma. Arq Bras Endocrinol Metabol. 2011;55:653-660.

11. Behrendt D, Ganz P. Endothelial function. From vascular biology to clinical applications. Am J Cardiol. 2002;90:40L-48L.

12. Bahia L, de Aguiar LG, Villela NR, Bottino D, Bouskela E. [The endothelium in the metabolic syndrome]. Arq Bras Endocrinol Metabol. 2006;50:291-303.
13. Hsueh WA, Lyon CJ, Quiñones MJ. Insulin resistance and the endothelium. Am J Med. 2004;117:109-117.

14. Heikens J, Ubbink MC, van der Pal HP, et al. Long term survivors of childhood brain cancer have an increased risk for cardiovascular disease. Cancer. 2000;88:2116-2121.

15. Colao A, Di Somma C, Rota F, et al. Common carotid intima-media thickness in growth hormone (GH)-deficient adolescents: a prospective study after GH withdrawal and restarting GH replacement. $J$ Clin Endocrinol Metab. 2005;90:2659-2665.

16. Meeske KA, Siegel SE, Gilsanz V, et al. Premature carotid artery disease in pediatric cancer survivors treated with neck irradiation. Pediatr Blood Cancer. 2009;53:615-621.

17. Bilora F, Pietrogrande F, Campagnolo E, et al. Are Hodgkin and nonHodgkin patients at a greater risk of atherosclerosis? A follow-up of 3 years. Eur J Cancer Care (Engl). 2010;19:417-419.

18. Gianicolo ME, Gianicolo EA, Tramacere F, Andreassi MG, Portaluri M. Effects of external irradiation of the neck region on intima media thickness of the common carotid artery. Cardiovasc Ultrasound. 2010;8:8.

19. Dullaart RP, de Vries R, van Tol A, Sluiter WJ. Lower plasma adiponectin is a marker of increased intima-media thickness associated with type 2 diabetes mellitus and with male gender. Eur J Endocrinol. 2007;156: 387-394.

20. Norata GD, Raselli S, Grigore L, et al. Leptin:adiponectin ratio is an independent predictor of intima media thickness of the common carotid artery. Stroke. 2007;38:2844-2846.

21. Wiesner P, Tafelmeier M, Chittka D, et al. MCP-1 binds to oxidized LDL and is carried by lipoprotein(a) in human plasma. J Lipid Res. 2013;54:1877-1883.

22. Brandalise S, Viana M, Pereira W, et al. Chemotherapy in 853 unselected childhood ALL patients. Results of the Brazilian multicenter trial GBTLI ALL-93 [abstract]. Pediatr Blood Cancer. 2004;43:s399.

23. Scrideli CA, Assumpção JG, Ganazza MA, et al. A simplified minimal residual disease polymerase chain reaction method at early treatment points can stratify children with acute lymphoblastic leukemia into good and poor outcome groups. Haematologica. 2009;94: 781-789.

24. Marshall WA, Tanner JM. Variations in pattern of pubertal changes in girls. Arch Dis Child. 1969;44:291-303.

25. Marshall WA, Tanner JM. Variations in the pattern of pubertal changes in boys. Arch Dis Child. 1970;45:13-23.

26. National Center for Health Statistics. Centers for Disease Control and Prevention Growth Charts. 2000. Available from: http://www.cdc.gov/ growthcharts/clinical_charts.htm.

27. Matthews DR, Hosker JP, Rudenski AS, Naylor BA, Treacher DF, Turner RC. Homeostasis model assesssment: insulin resistance and beta-cell function from fasting glucose and insulin concentrations in man. Diabetologia. 1985;28:412-419.

28. Urbina EM, Williams RV, Alpert BS, et al; American Heart Association Atherosclerosis, Hypertension, and Obesity in Youth Committee of the Council on Cardiovascular Disease in the Young. Noninvasive assessment of subclinical atherosclerosis in children and adolescents: recommendations for standard assessment for clinical research: a scientific statement from the American Heart Association. Hypertension. 2009;54:919-950.

29. Dengel DR, Kelly AS, Zhang L, Hodges JS, Baker KS, Steinberger J. Signs of early sub-clinical atherosclerosis in childhood cancer survivors. Pediatr Blood Cancer. 2014;61:532-537.

30. Stabe C, Vasques AC, Lima MM, et al. Neck circumference as a simple tool for identifying the metabolic syndrome and insulin resistance: results from the Brazilian Metabolic Syndrome Study (BRAMS). Clin Endocrinol (Oxf). 2013;78:874-881.

31. Ciccone MM, Bilianou E, Balbarini A, et al. Task force on: 'early markers of atherosclerosis: influence of age and sex'. J Cardiovasc Med (Hagerstown). 2013;14:757-766.

32. Reilly JJ, Kelly A, Ness P, et al; ALSPAC Study Team. ALSPAC Study Team: premature adiposity rebound in children treated for acute lymphoblastic leukemia. J Clin Endocrinol Metab. 2001;86:2775-2778. 
33. Wildman RP, Janssen I, Khan UI, et al. Subcutaneous adipose tissue in relation to subclinical atherosclerosis and cardiometabolic risk factors in midlife women. Am J Clin Nutr. 2011;93:719-726.

34. Demerath EW, Reed D, Rogers N, et al. Visceral adiposity and its anatomical distribution as predictors of the metabolic syndrome and cardiometabolic risk factor levels. Am J Clin Nutr. 2008;88:1263-1271.

35. Fagan TC, Deedwania PC. The cardiovascular dysmetabolic syndrome. Am J Med. 1998;105:77S-82S.
36. Dawson JD, Sonka M, Blecha MB, Lin W, Davis PH. Risk factors associated with aortic and carotid intima-media thickness in adolescents and young adults: the Muscatine Offspring Study. J Am Coll Cardiol. 2009;53:2273-2279.

37. Jarfelt M, Lannering B, Bosaeus I, Johannsson G, Bjarnason R. Body composition in young adult survivors of childhood acute lymphoblastic leukaemia. Eur J Endocrinol. 2005;153:81-89.

\section{Publish your work in this journal}

Vascular Health and Risk Management is an international, peerreviewed journal of therapeutics and risk management, focusing on concise rapid reporting of clinical studies on the processes involved in the maintenance of vascular health; the monitoring, prevention and treatment of vascular disease and its sequelae; and the involvement of metabolic disorders, particularly diabetes. This journal is indexed on PubMed Central and MedLine. The manuscript management system is completely online and includes a very quick and fair peer-review system, which is all easy to use. Visit http://www.dovepress.com/ testimonials.php to read real quotes from published authors. 